\title{
Finding the Relationship Between News and Social Media Users' Emotions in the COVID-19 Process
}

\author{
(D) Ahmet Anıl Müngen ${ }^{1}$, Dìrfan Aygün², (D) Mehmet Kaya ${ }^{3}$ \\ ${ }^{1}$ Department of Computer Engineering, Firat University, Elazığ, Turkey; amungen@gmail.com; \\ 2 Department of Software Engineering, Celal Bayar University, Manisa, Turkey; irfan.aygun@cbu.edu.tr; \\ ${ }^{3}$ Corresponding Author; Department of Computer Engineering, Frrat University, Elazı̆̆ Turkey; \\ kaya@firat.edu.tr;
}

Received 09 September 2019; Revised 18 November 2020; Accepted 24 November 2020; Published online 30 December 2020

\begin{abstract}
Nowadays, social media and online sharing sites are frequently used to share thoughts about daily events. Thanks to the posts made by internet users on these platforms, first, quite big data is generated to interpret the agenda. More than 10,000 comments of more than 5000 users made about COVID-19 from online websites between 15 March and 15 May were collected in this study. Then, emotional analysis on these comments was carried out with BERT, GRU, LSTM and TF-IDF methods. The changes in the amount of user comments and the emotions reflected by the comments have been associated with the actual events of these dates. It has been determined which types of events affect users more. In addition, the emotional response changes of the users to the official COVID19 statistics were measured and the peak points of the emotional changes were determined. Finally, the emotion classification methods applied were evaluated by user questionnaires and their successes were determined according to F-Measure.
\end{abstract}

Keywords: COVID-19, LSTM, BERT, Sentiment Analysis, TF-IDF

\section{Introduction}

Social networks are virtual interaction models that reflect the interests, friendships and connections of users in their real life. Social media adventure on the internet started with people making their own news. Today, it is seen that all agencies, including major news organizations, use these platforms to broadcast their news. Social media hosts many different types of news, as it is both a news outlet and a space for users to share their own news. Users access the news on social media and can convey their reactions and comments to the news via social media. Naturally, social networks have become the priority sharing areas where people share their reactions to instant events.

Even though social networks are very popular platforms for sharing user ideas, there are also alternative websites preferred by users. These websites, which were called "forum" in the past, are more systematically constructed than social media. They are divided into specific categories and contain longer comments within sub-titles. Although the history of the forums reached the 1990s, there are still quite popular forums that are actively used today. As an example, Reddit is one of the most popular forums with its subject and category-based sharing. Unlike social networks, in the forums, users make their opinions not on their own pages, but inside the page opened for a topic. This form of sharing provides a ready-made database of subject-oriented comments for research on a topic.

Associating the comments collected from social networks with current topics and making analyzes from comments on a topic is used frequently in academic studies. Especially for researchers working on data mining, these forums are like a database. In this study, the effects of events related to COVID-19 pandemic on users were investigated with data collected from social networks. Changes of emotions were determined according to real events by analyzing with user comments, data mining and deep learning methods. It is aimed to identify the factors that affect the society in the pandemic process by associating the emotional changes obtained with the events that took place at that time.

The rest of the paper is organized as follows: Section 2 is devoted to the discussion of related work. Section 3 describes the processes related to the collection of data used in selected social networks. In 
Müngen et. al

Section 4, the method proposed in this study is first mentioned and then the experimental results for the proposed method are given. Section 5 concludes the paper.

\section{Related Work}

Sentiment analysis in comments on websites and social networks is a field that is subject to academic studies in many languages for different topics with different algorithms. Sentiment analysis can be applied at four different levels. These levels are sentence, status, document and user level, respectively. Different methods can be applied within these four levels, such as machine learning, lexicon, NLP, ontological or hybrid solutions [1].

Many studies have been conducted on the detection of messages that are described as spam or false messages on social networks [2] [3]. Sentiment analysis has also been used to analyze advice processes for brands and services [4]. Sentiment analysis processes have also been used frequently in big data and are frequently used in commercial projects [5]. It has been revealed in many studies that sentiment analysis can be used not only in canonical texts, but also in an uncorrected / unstructured spelling language, which is the language most often used by users [6].

In their study, Anjaria and Guddeti conducted emotional analysis on brands, people and events using different algorithms on Twitter social network [6]. Many researchers have previously done work for Weibo [7] [8] in the Chinese Language. Nehri et al did the same work for the Italian RA1 media broadcaster in the Italian language [9]. In their study, Contrantes et al proposed a method aimed at solving the problem of starting cold on a new product via Twitter and Facebook [10]. Tang et al. conducted a study demonstrating the importance of emotions in purchasing and measuring the impact of social network data and physically sold products on users [11].

Sentiment analysis can be performed at different levels [12]. First level can be defined as algorithms that perform sentiment analysis on sentence basis. The second level methods are document-based and look at all the sentences in the document to see if the comment is completely or partially positive / negative. The third level is subject-based sentiment analysis. Each subject / word can stand out from a different emotion aspect. For example, in the sentence "this computer is very good and quick, but it is running out of battery very quickly", positive and negative comments were made for the computer. In a computer-based analysis, the sentence will be positive but in a battery-based analysis it will be negative. In this type of work, all emotion is not connected to a single object.

There are also different application methods in terms of the algorithm to be used in sentiment analysis. The word-based tagging system is the most principal of these algorithms [13]. The second method is those using NLP [14], Lexicon [15] or machine learning [16]. The third method performs sentiment analysis by comparing it with a trained data set [17]. In addition, methods that perform positive / negative information analysis at the user level have also been proposed [18]. Analysis of the effects of users on social networks on other users is one of the studies used with these methods [19].

The Ekşi Sözlük user-based Turkish forum, which we selected as a data set, has been the subject of many academic researches before. Alp has analyzed users discourses on one of the ethnic groups in Turkey [20]. Doğu et al. conducted a study on Ekşi Sözlük concept of authorship [21]. Akınerdem analyzed the current TV series by comparing the comments in Twitter and Ekşi Sözlük [22]. Almost all of the work done with Ekşi Sözlük focuses on sociological and / or psychological analysis.

Although it is a very new subject yet, academic studies on COVID-19 focused social media interaction have started. Depoux et al. [23] examined the reflection of human panic on social networks after the COVID-19 news. Gao et al. [24] analyzed the increasing mental health problems after COVID-19 through social media. Li et al. analyzed the increase of COVID-19 web inquiries over search engine data [25]. Pennycook et al. [26] investigated methods of preventing misinformation spread over social networks related to COVID-19. Li et al. [27] tried to determine the speed and types of spread by classifying the information published on social media during the COVID-19 process. 
Müngen et. al

\section{Data Collection}

In this study, the reactions of the users to real events were tried to be collected from the messages collected from web-based forums. An event series that occupies the agenda and where many people comment is selected. Due to the impact of the COVID-19 pandemic on both our individual life and its social impact, the agenda for the virus was selected in our study [28]. Although the widely used social media platforms (Twitter, Instagram, etc.) contain a lot of data, it was not deemed suitable as a dataset since this data is short and contains a lot of spam. Although the COVID-19 virus has affected the whole world, it is necessary to focus on a limited time and a limited area in order to find the link between real events and people's emotions. The study focuses on Turkish-speaking users in Turkey from 15 March to 15 May. Ekşi Sözlük which is Turkey's largest forum and one of the most frequently visited site is selected for user reviews, user information, and scores given to comments by users. Data collection process was carried out through a special bot within the scope of this study.

Ekşi Sözlük is a common "dictionary" based on the concept of websites built on user contribution. Registered members send contents to the site, such as articles, links, text messages and pictures, which are then interpreted and / or rated by other members. Different pages and topics were created by users on thousands of topics such as current news, science, movies, video games, music, books. Currently with more than 400,000 registered users, including 100,000 authors it is of one of Turkey's largest online community. Ekşi Sözlük offers a platform for sharing information on various topics from scientific issues to daily life by thousands of people, as well as a virtual socio-political platform for discussing current political content and sharing personal opinions [29].

Since an analysis of COVID-19 will be done in the study, the data are taken from the pages in this topic. All comments in the titles created in the last 2 months related to COVID-19 were recorded together with the author information and number of likes in order to create a data set. As the first step of data processing, short comments that contain only links were not excluded. Also, only comments with emoji have been removed from the dataset.

To compare the data collected, the daily number of deaths and infected patient's information was collected from the Republic of Turkey Ministry of Health webpages. All comments and ministry data are noted daily. In addition, the events to be used for impact value measurement in our study were selected from the most frequent agenda events in the news. Some information about the collected data is shown in Table 1.

Table 1 Collected Data

\begin{tabular}{ll}
\hline Dataset & Number \\
\hline Total Comment & 17903 \\
\hline Number of Different Authors & 7834 \\
\hline Number of Likes & 186999 \\
\hline
\end{tabular}

The intensity of the comments collected by days is shown in Figure 1. In January and February, in contrast to the World, Covid-19 cases have not been observed in Turkey, so the number of comments is very small. Although there are a few peak points starting from the second week of March, it is seen that there is a great density in the third and fourth week. In April, similar intensity comments continued, except for 2 peaks. In May, with the reduction of the impact of the pandemic in Turkey, the amount of comments had begun to fall. 


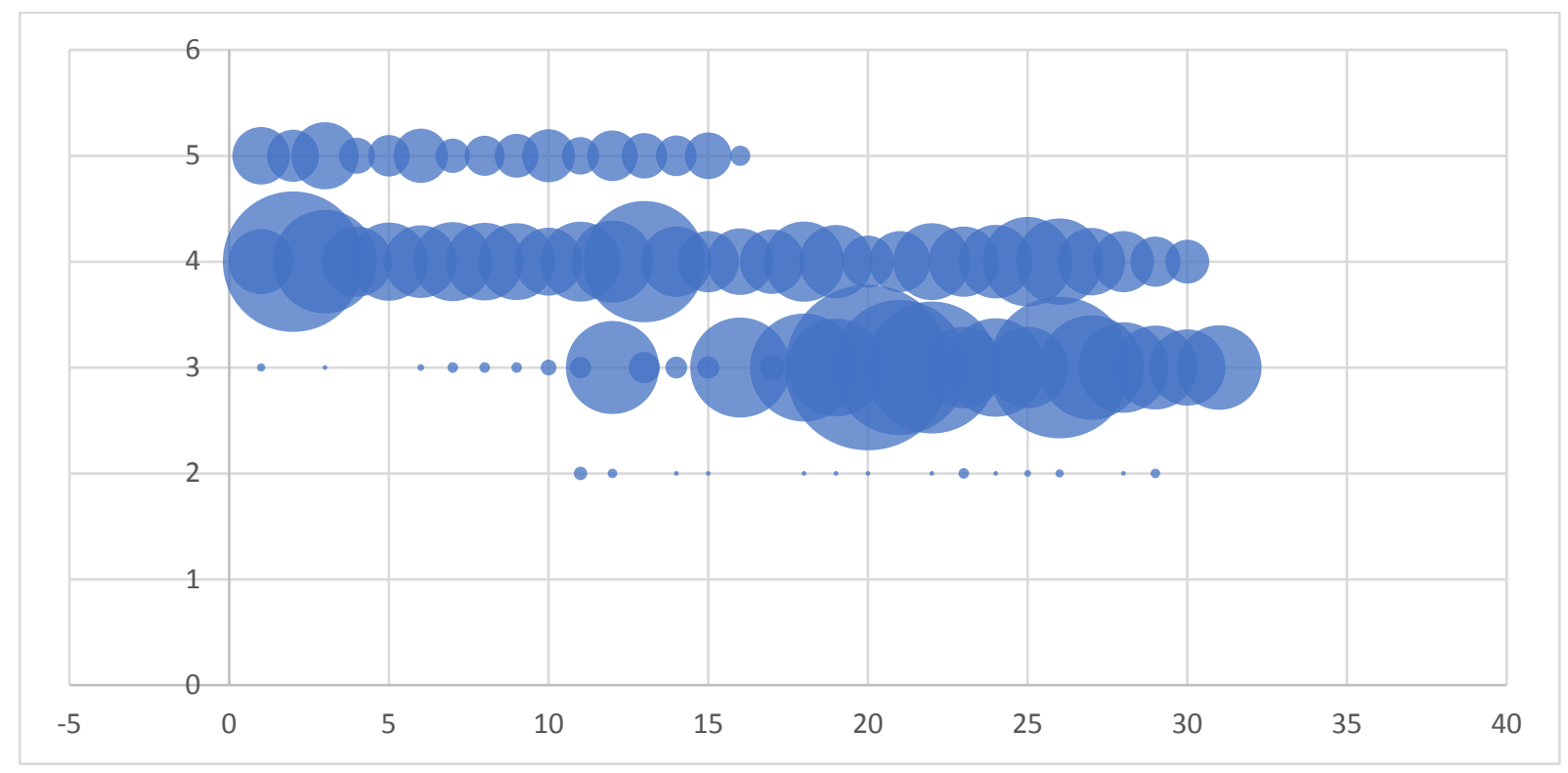

Figure 1 Density Map of Comments by Days

When choosing important events, the most commented days were first found to have a social response. When calculating the days with the most comments, the requirement for the number of comments per day to be at least $25 \%$ higher than the average of the 5 days before that day was checked. The days selected in accordance with this condition are shared in Table 2 . The events related to the days were chosen among the most talked events in that day. Table 2 is determined for 8 days and events related to each day are listed.

Table 2 Dates of Increasing Comments and Significant Events

Date Event $\quad$ Topic

12 March Under President Erdogan's chairmanship, a 5-hour coronavirus meeting Sports, was held. Sports competitions have been postponed, and some Travel, preventions have been taken regarding overseas departure. Primary Agenda schools were closed for 1 week and universities for 3 weeks [30].

16 March Turkish Health Minister Fahrettin Koca said Europe and the Middle Agenda East based, new 29 coronavirus patients were discovered in Turkey. Since there were 18 cases in total in previous days, the number of cases increased close to $150 \%$. [31]

18 March Some items from the economic package of 100 billion TL announced Economy on March 18

20 March All hospitals were declared as pandemic hospitals, both the number of Agenda cases and deaths increased by $100 \%$ from the sum of the past days. [31]

\begin{tabular}{llc}
\hline $\mathbf{2}$ April & Infected patient numbers by cities was announced. & Agenda \\
\hline $\mathbf{1 3}$ April & '’When Does COVID-19 End?’ Virtual Discussion Started & Agenda \\
\hline $\mathbf{1 8}$ April & $\begin{array}{l}\text { Turkey's infected patient number passes its neighbor Iran and Turkey } \\
\text { rises to the eighth place in the infected patients list in the world }\end{array}$ & Agenda \\
\hline $\mathbf{2 5}$ April & $\begin{array}{l}\text { TÜBİTAK President Gave the Release Date for Coronary Virus } \\
\text { Vaccine }\end{array}$ & Agenda \\
& &
\end{tabular}


While selecting events for Table 2, the most spoken and most influential event of each category was noted. In addition, 4 events that take up a lot of space in the news agenda but are not included in important dates are shown in Table 3. Later in the study, there will be relations between the events in these tables and the reactions of people.

Table 3 The Events Which Did Not Get Much Comments

\begin{tabular}{lll}
\hline Date & Event & Topic \\
\hline $\mathbf{2 0}$ March & $\begin{array}{l}\text { Closing of all places of worship by the Directorate of Religious } \\
\text { Affairs }\end{array}$ & Religion \\
\hline $\mathbf{2 3}$ March & $\begin{array}{l}\text { Fatih Terim who is Coach of Galatasaray which is one of Famous } \\
\text { Turkish football club was announced Covid-19 Positive. }\end{array}$ & Sport \\
\hline $\mathbf{2 5}$ March & $\begin{array}{l}\text { All public and private schools were declared a vacation for over } \\
\text { one month. }\end{array}$ & Education \\
\hline $\mathbf{2 6}$ March & $\begin{array}{l}\text { All universities were declared a vacation by the Higher Education } \\
\text { Institution }\end{array}$ & $\begin{array}{l}\text { High } \\
\text { education }\end{array}$ \\
\hline
\end{tabular}

\section{Methodology And Experimental Results}

In this section, the results obtained from the methodology and experiments used to interpret the data are examined. Owing to the obtained research results, the successes of the methods used in the experiments are shared comparatively.

All the experiments shared in this section were carried out in Windows 10 environment with a computer with 16 GB RAM and $2.5 \mathrm{GHz}$ processor power. Python-3 was used as the programming language in the experiments. Spyder and Jupyter infrastructure provided with Anaconda were used as the development platform. In addition, Tensorflow, Keras and Numpy libraries with open source code were used to provide ease of operation in the experiments.

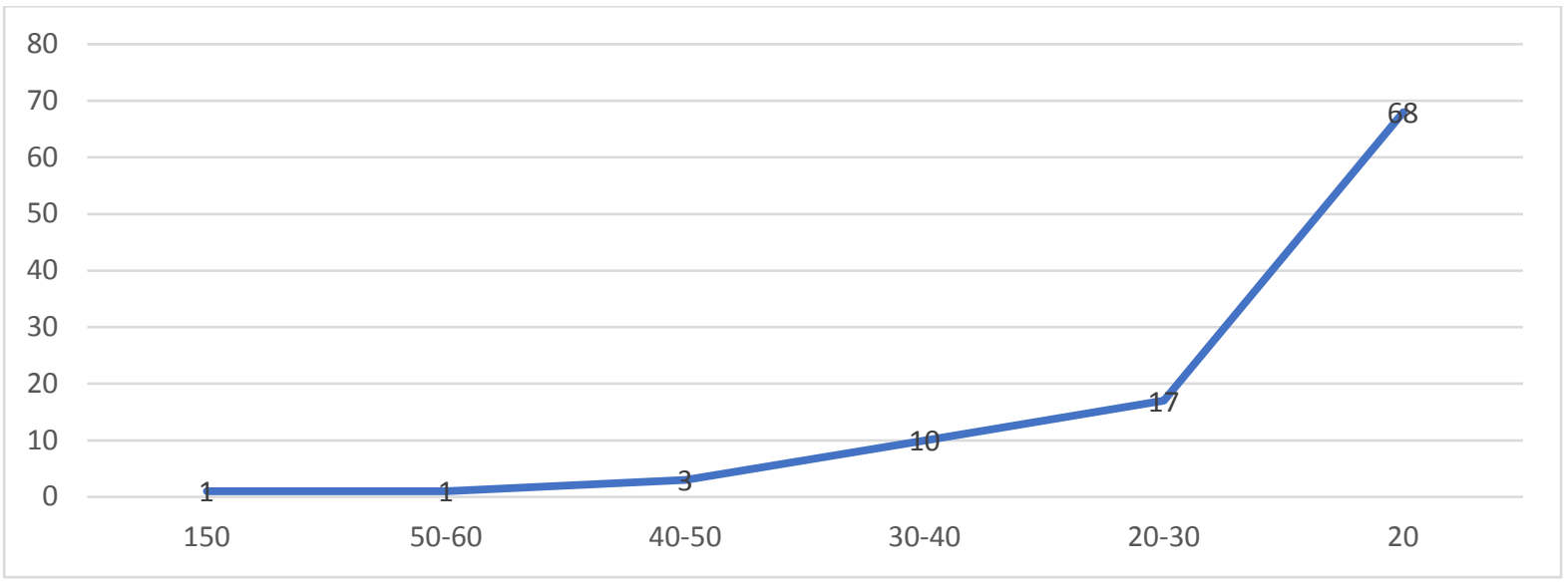

Figure 2 The Density Graph of the 100 Most Commented Users

The 100 most commenting users and their frequency of commenting are analyzed in Figure 2. The number of users with more than 150 comments is only one. The number of users who comment between 50 and 150 is one. The number of users who comment between 40 and 50 is three. The number of users who comment between 20 and 30 is 17. The number of users who comment between 10 and 20 is at least 68. Most users commented less than 10 times. When a 60-day period is taken, it is normal to make 10 or fewer comments. In this context, it is evaluated that the data is not collected for same users and that it is collected from sufficiently different users. At the same time, it will be possible to examine emotional change over time with ten or more comments. Figure 3 shows the density chart of the 10 most commented users. 


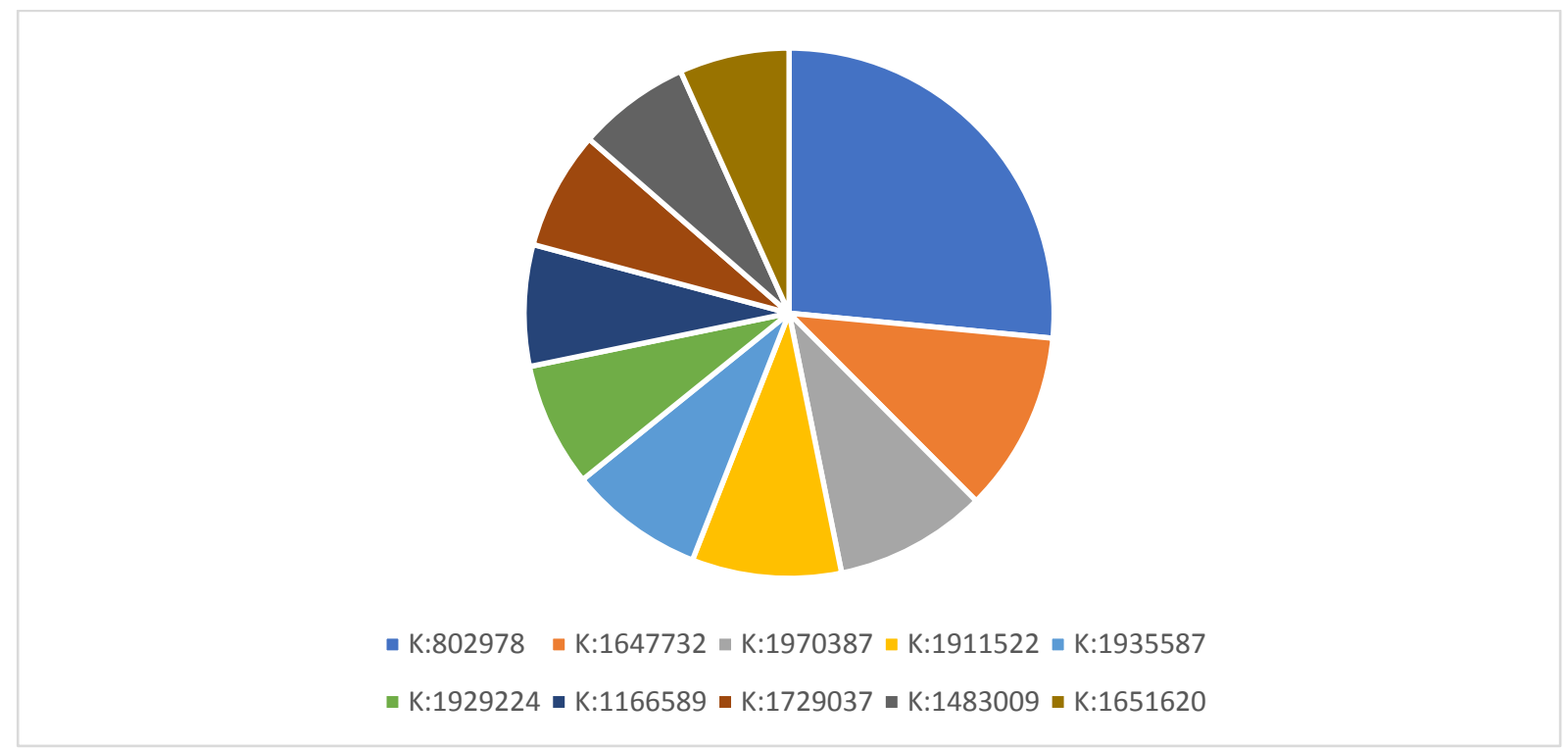

Figure 3 The Density Chart of the 10 Most Commented Users

When the figure is evaluated, it is seen that no user has made enough comments to manipulate the data. Although the user who comments most frequently in this chart seems to occupy nearly $30 \%$, this is only the chart among the top 10 users who comment most frequently. Looking at the whole chart, even the most frequent user takes up less than $1 \%$ of the total data. The impressions on the chart that begin with 'K:' are the id's obtained after anonymizing the user information. The number of comments made by users according to months is shared in Figure 4.

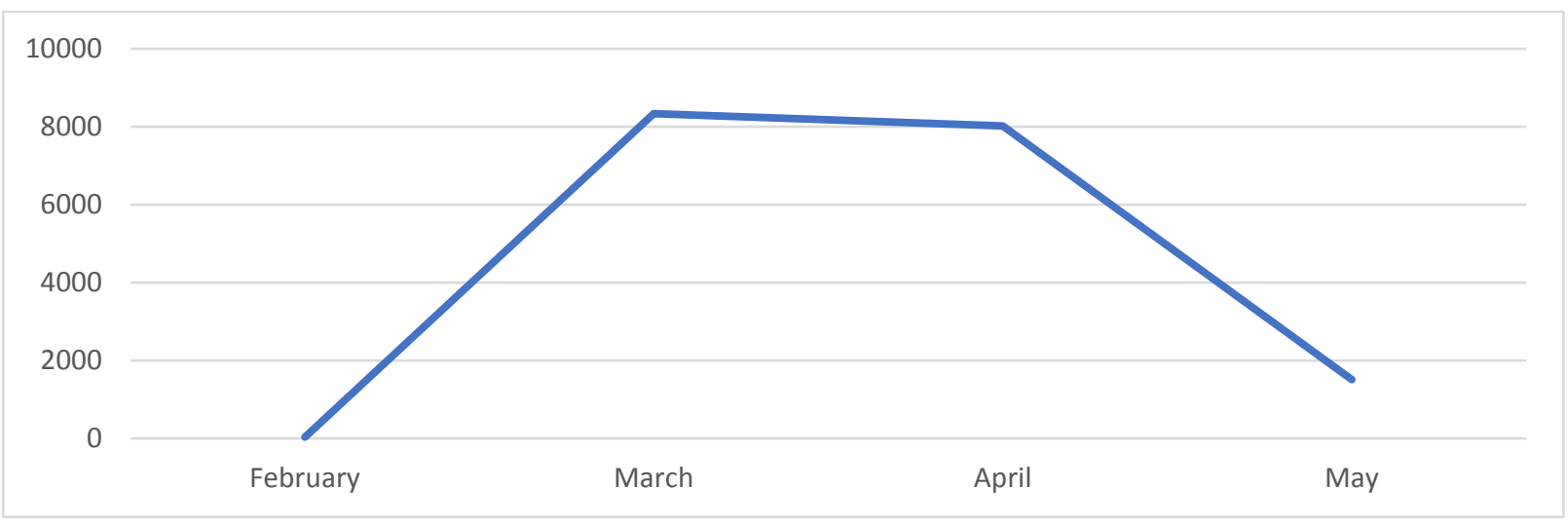

Figure 4 Frequency of Commenting by Months

When the chart is analyzed, it is seen that there are very few comments in February compared to other months. It was observed that the comments peaked in March and April. Although only the first 15 days of May were taken, it is seen that the level of March and April will not be reached in May, according to the average of the current days. In Figure 5, the number of comments is shared by weeks, with a more focus on.

When the distribution of the data according to the weeks of the year is examined, it is seen that the users make the most intensive comments in the 11th and 12th weeks. The concentrated part of the data shows the last two weeks of March. Major events seen in Table 2 also generally occurred this week. When the weeks are examined, it is obvious that the first two weeks of March have passed with very few comments and the main intensity is in the last 2 weeks, and the high of the whole month is due to the intensity in these 2 weeks. The relationship between the comments and the number of cases was measured with 2 different types of graphics. The first of these graphs is the coefficient graph and is shown in Figure 6. 


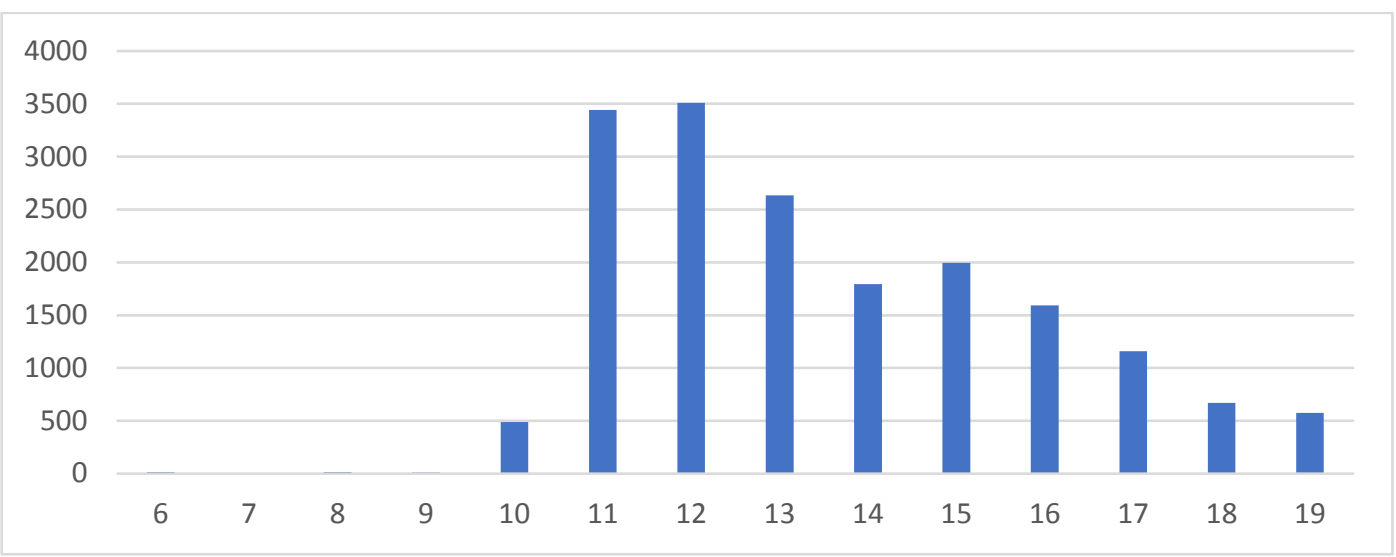

Figure 5 Frequency of Commenting by Weeks

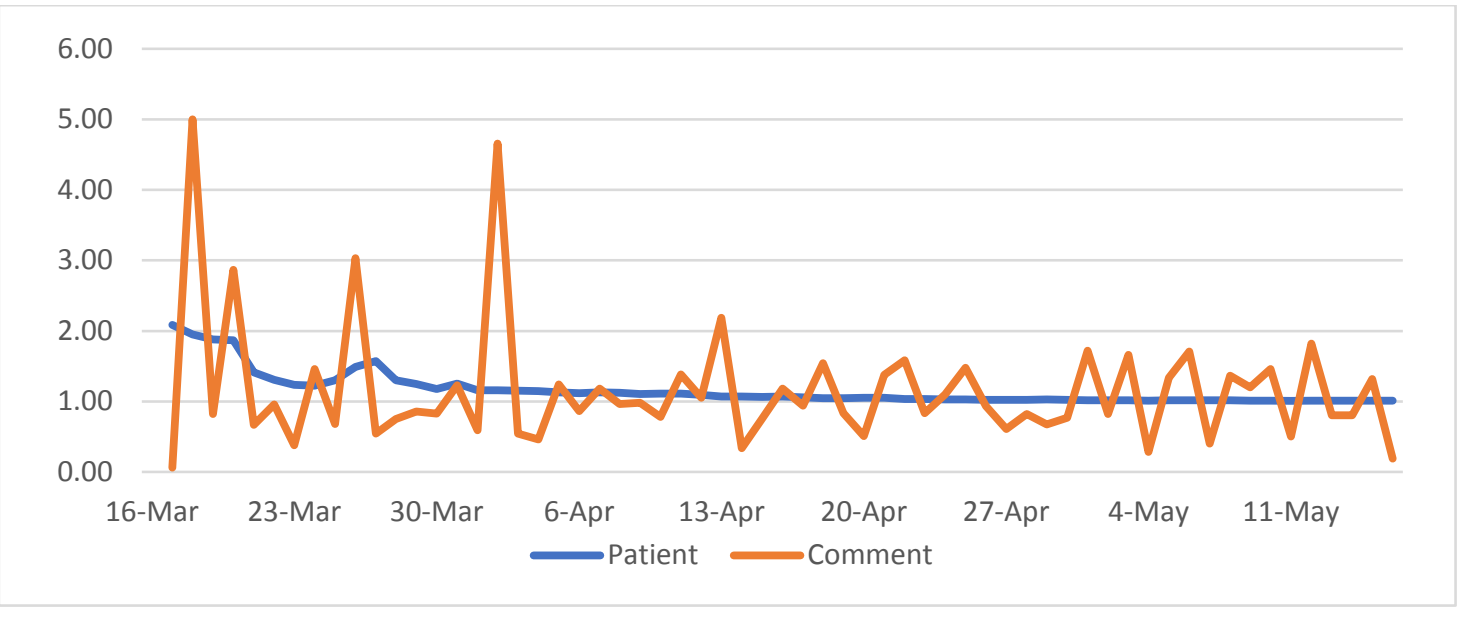

Figure 6 Comparison Chart of Case and Comment Coefficients by Days

In the coefficient graph, all numbers are taken by days and the changes are calculated to show how many times it is bigger than the previous day. In this way Sudden changes were tried to be detected. As the reason for the sudden change on March 18, it can be seen that there were very few comments in the previous days. However, it is still seen as a more realistic reason that the change is related to the economic package announced at this date. Changes in the March 20 can be explained by the news of the turning of all hospitals in Turkey to pandemic hospitals. The increase in the number of patients on the 18th and 20th March continues nearly twice. The increase in the number of patients on March 26 is also the regional peak point and on the same days, a peak was identified in the comments.

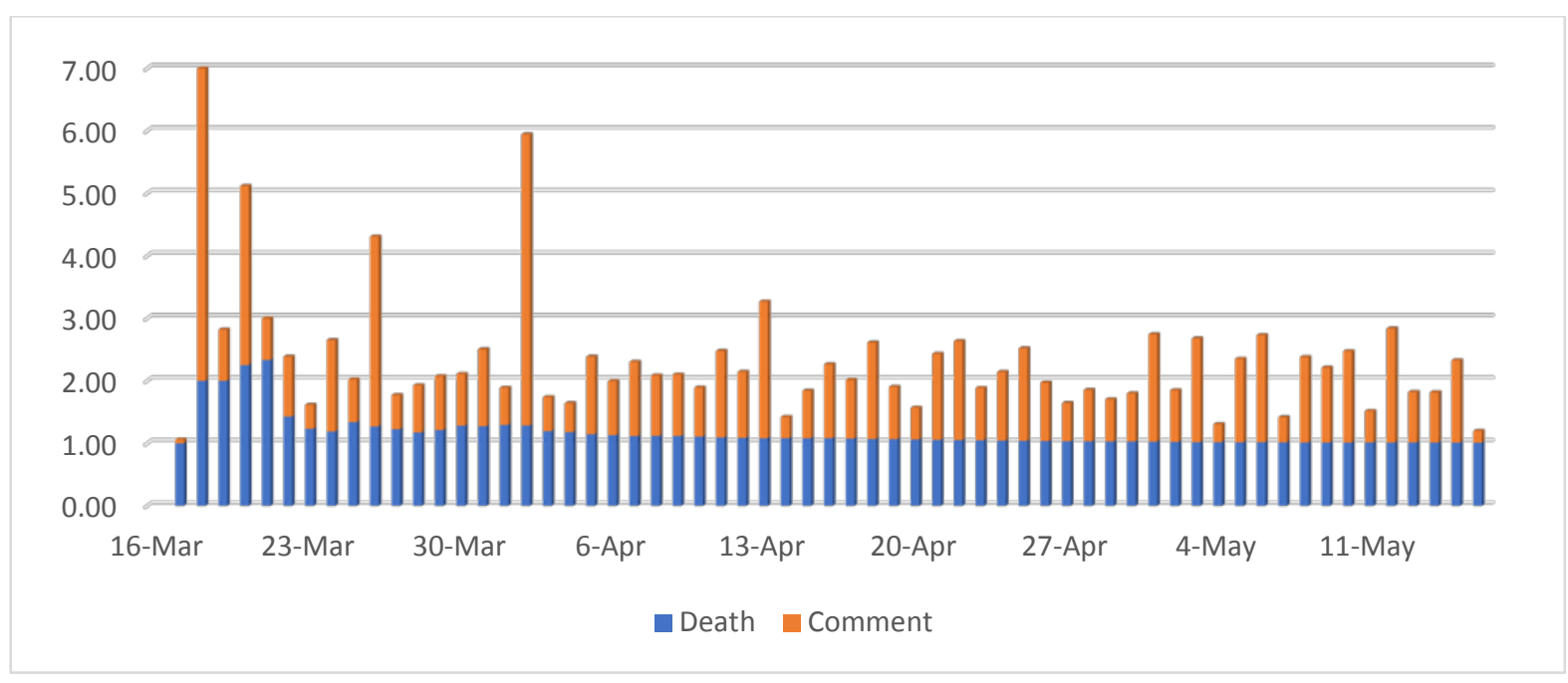

Figure 7 Death and Interpretation Coefficient Completion Chart by Days 
The relationship between the coefficient of death and the coefficient of interpretation shown in Figure 7 shows parallelism at many peak points in the table. It is seen that many external variables expressed in the previous table cause other changes. The connection between the number of deaths and the number of comments is thought to be more proportional to the number of cases.

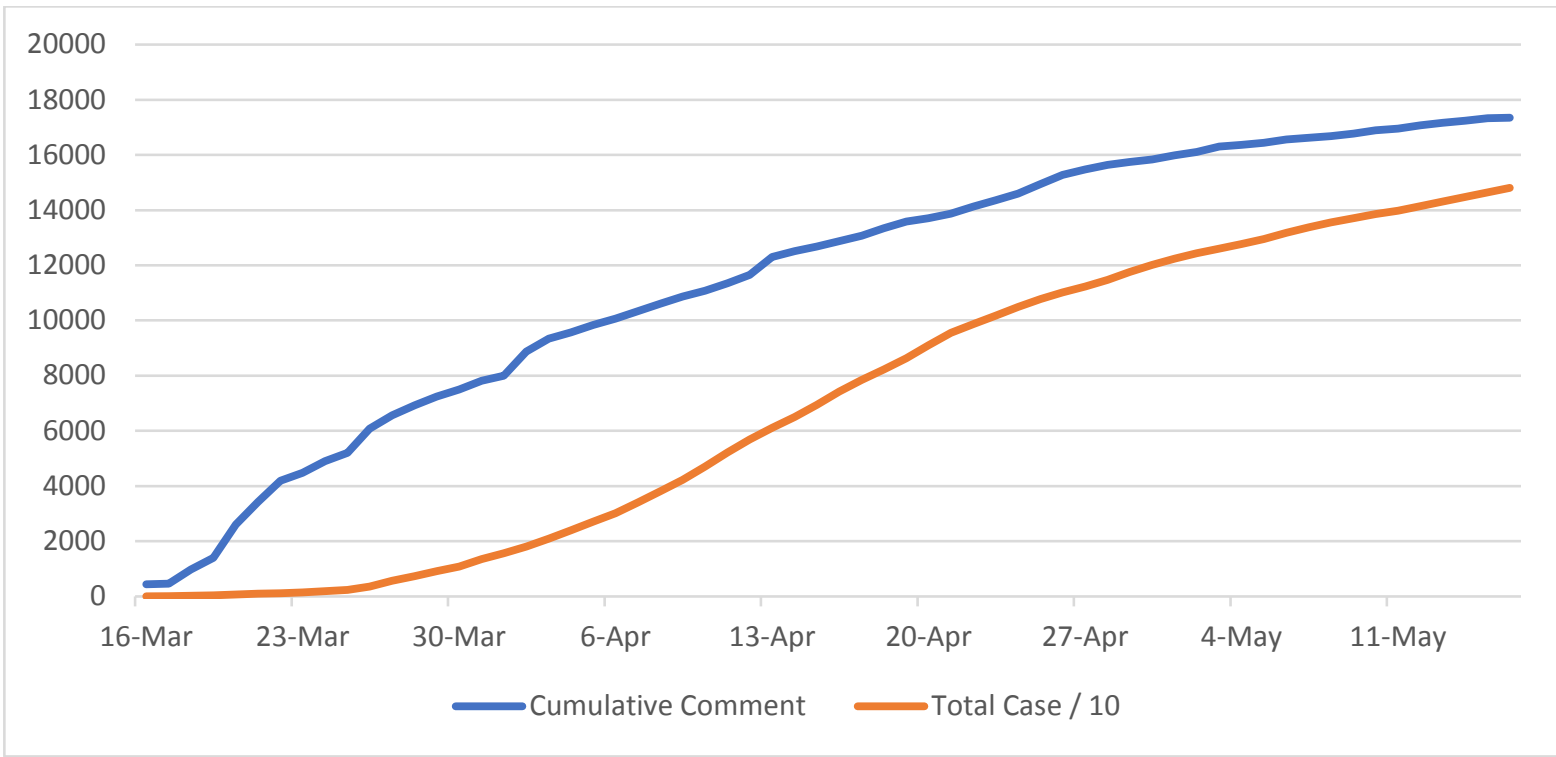

Figure 8 Cumulative Data Graph of Death and Interpretation by Days

In the graph in Figure 8, unlike the previous two graphs, the cumulative sums, not the coefficients, were handled. When evaluated cumulatively, it is seen that the comments entered the sudden rise much earlier than the cases. It is also observed that it captures the linear point earlier and then increases with a smaller curve. The number of cases started to peak approximately 20 days after the number of comments and caught a curve parallel to the number of comments. Although the number of comments increased suddenly to pass to the linear curve within 10 days, the number of cases increased in approximately 20 days and then switched to the linear curve.

The table shows that people more strongly respond to cases but get used to it in time. When the number of cases peaked, small peaks were observed in the number of comments, but it was observed that the case did not increase at the same rate as the rate of increase.

Sentiment analysis of all collected comments was determined by four different methods. One of these methods is the method of labeling with TF-IDF [32]. With this method, the positive and negative words in the comments are tagged and then the frequency count is made and the comment is determined as positive, negative or neutral. $75 \%$ of all tagged words were expected to have the relevant tag to identify a comment as positive or negative. With this rule, comments with a confidence value less than $75 \%$ are marked neutral. Tags are made by identifying 50 most common positive and 50 most common negative words in Turkish language.

As a second method is supervised LSTM [33]. As the training data, the results accepted by unanimous or majority vote from 34.397 classified comments in the TREMO [34] data set were used. The labels in this training data have been converted as positive, negative and neutral. A second LSTM test was performed using previously trained Word2Vec [35] vectors. Thanks to this method, it is aimed to produce more successful semantic results for words that the model does not encounter in education. Unlike standard LSTM architecture, inputs consisting of word vectors are used. A data set of Turkish texts [36] and the TREMO data set were combined to form word vectors. Then, each word vector created was sent as an input to LSTM and aimed to produce more successful semantic results.

The third method is the advanced Recurrent Neural Network (RNN) structure called the Gated Recurrent Unit (GRU) [37]. It is known that the use of GRU increases the success rate in many classification problems and uses less resources compared to LSTM [38]. In the experiments, it was observed that the GRU model increased the success (acc) from 0.84 to 0.91 according to the LSTM model using the same 
training set. Therefore, in addition to other methods, the results obtained with GRU are also shared in the study. While creating the model, embedding layer was added before GRU layers and word vectors were used as input. The resources used in the LSTM method were used in the training of the model and the creation of word vectors.

BERT [39] algorithm is used as the last test method. This algorithm stands out from other word embedding techniques thanks to its transfer learning approach and pre-trained neural networks. Thanks to the bidirectional working logic, it has become possible to extract the vector of a word by evaluating the previous and subsequent sentences. Along with this method, it has been observed that it significantly increases the success in the studies to make sense from the text [40]. In experiments with BERT, the same training content as the GRU experiment was used for training.

The reason why the neutral number is too high is because the short comments are mostly labeled as neutral. Since the short comments are not evaluated at all or labeled as neutral, they are labeled as neutral as they are the same for the evaluation phase. Also, in LSTM / GRU experiments, the result of the prediction is generated between 0 and 1 thanks to a sigmoid. As these values approach 1 , the result is understood to be positive, and as it gets closer to 0 , it is understood to be negative. In this way, since the value of 0.5 is in the middle, the comments between the values of $0.45-0.55$ seen close to this value are accepted as neutral.

When the data used in the study is examined; it is seen that the words without grammatical patterns, irregular words and words used in the daily speaking language which are not in the dictionary are frequently used. Although all these words are positive / negative, it makes labeling difficult and lowers the success rate. The sloppy use of language among young people and the transformation of the internet jargon over the years made it difficult to work.

In the study, in order to measure the success of four different methods, emotion analysis was carried out by survey participants on 1000 data randomly selected from all data. Success criteria were revealed by comparing the results with the results of the selected algorithms. Success results are shown in Table 4. As seen in previous academic studies, LSTM has been more successful in labeling than other methods. With this test, it was seen that all four methods used in the experiments were successful enough to be used in this study.

Table 4 Success Rates of Applied Methods in Emotion Analysis

\begin{tabular}{lcccc}
\hline & $\mathbf{A}$ & $\mathbf{P}$ & $\mathbf{R}$ & $\mathbf{F}$ \\
\hline Labeling with TF-IDF & 0.769 & 0.758 & 0.690 & 0.763 \\
\hline LSTM & 0.875 & 0.897 & 0.820 & 0.886 \\
\hline GRU & 0.817 & 0.893 & 0.770 & 0.854 \\
\hline BERT & 0.823 & 0.867 & 0.760 & 0.844 \\
\hline
\end{tabular}

It is also seen in these test results that there are positive and negative users in all conditions. Despite this, the peak points of emotion-intensive comments for the majority of users were determined to be on March 21 - March 26 and April 3. As a result of associating each peak with an event, it is concluded that the following events are directly related to the peak points.

The number of patients and the number of deaths is connected with COVID-19 in Turkey has increased by $100 \%$ on March 21 . This day is the first day for a big society response. When the first 1000 cases were ignored, March 26 was the first day with the highest incidence of infected patients in COVID-19. It is obvious that the sudden increase in the patient number and the number of patients exceeding 3000 caused anxiety for the users. The positive density on April 3; can be relevant with the explanation of the city-based Covid-19 infected number of patients in Turkey, the fact that Istanbul host the half of all infected cases in Turkey and the small amount of infected in other cities. These statistics coincide with the saying of "Istanbul can be Turkey's Wuhan" which is belong to The Minister of Health of the Republic of Turkey [41]. This supports the accuracy of other data. 


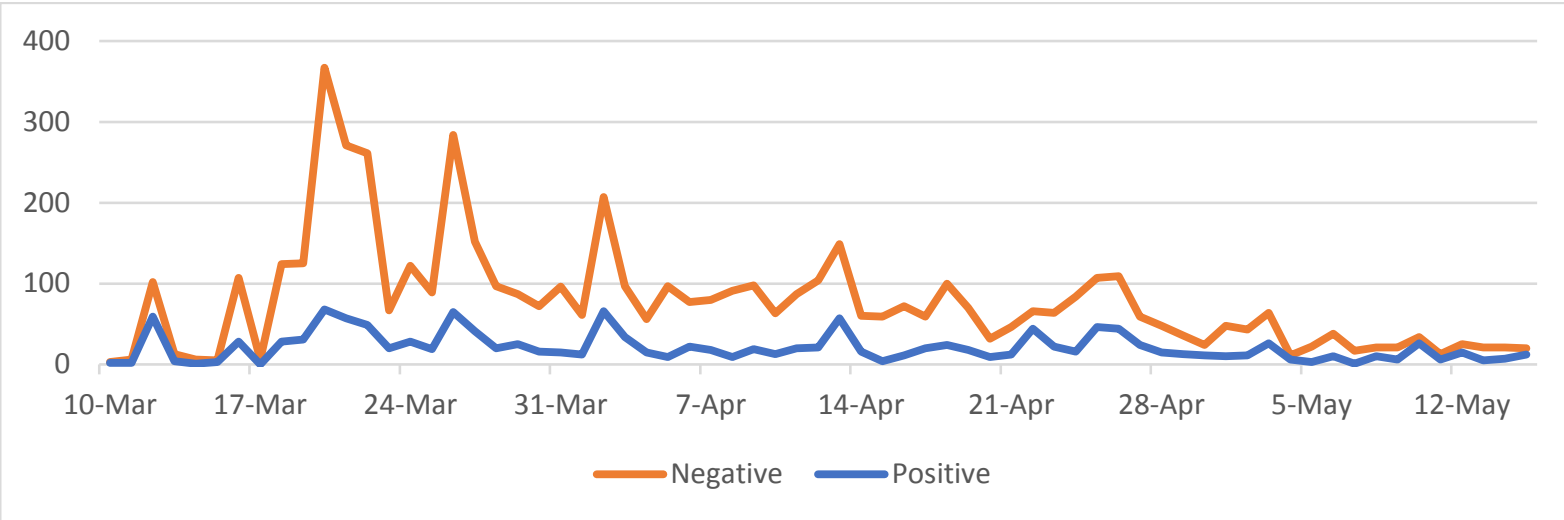

Figure 9 Distribution of Positive and Negative Comments by Days with TF-IDF Labeling Method

Figure 9 shows the graph of the number of comments with emotion analysis. From the test results, it is seen that when users have very negative or very positive emotions, they tend to write more comments than neutral emotions. It is also seen that negative emotions encourage writing more than positive emotions. The increasing level of negative comments in peak dates, shows people's anxiety. Positive comments are estimated to be users who try to think positively and are not in a state of panic.

Figure 10 shows the graph of the number of comments with emotion analysis. It is estimated that the reasons for the increase of positive comments on peak dates are not to make people panic and try to think positively. On the other hand, there was an increase in negative comments during these dates. Unlike the labeling method, LSTM has found a peak date for April 13 and April 25. It is seen that the comments made on April 25 have increased due to the press releases of state institutions related to vaccination studies. In the tests carried out with different methods in Figure 11 and Figure 12, similar results were obtained with the tests previously applied.

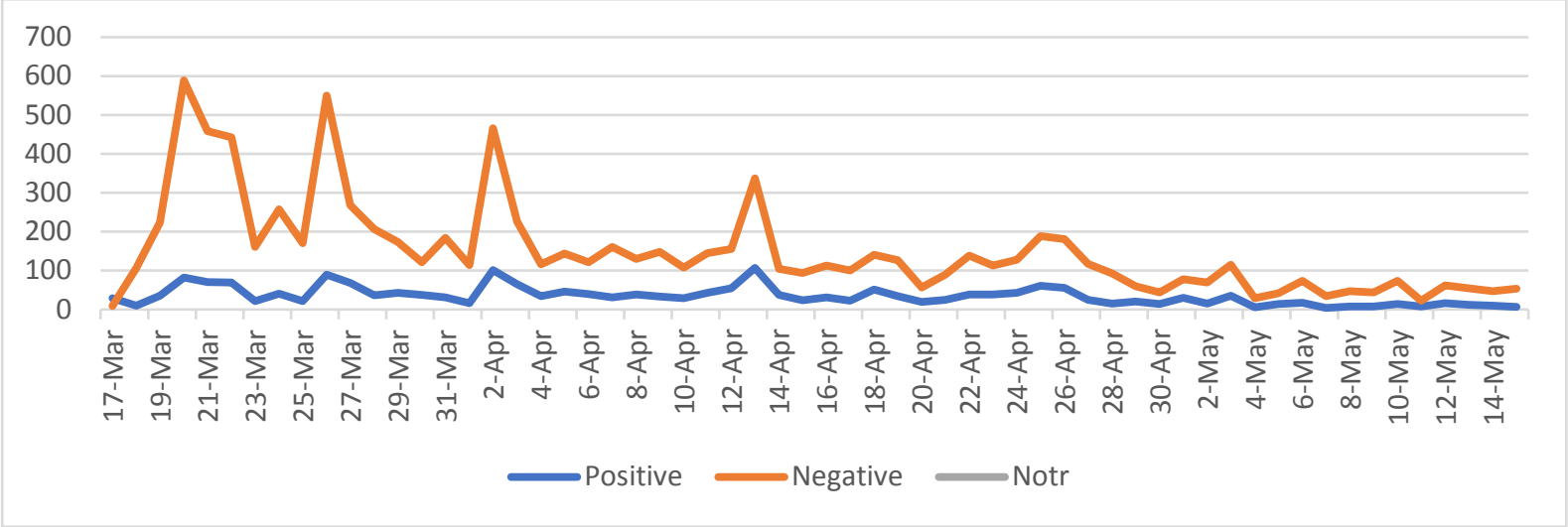

Figure 10 Distribution of Positive and Negative Comments by Days with LSTM Labeling Method

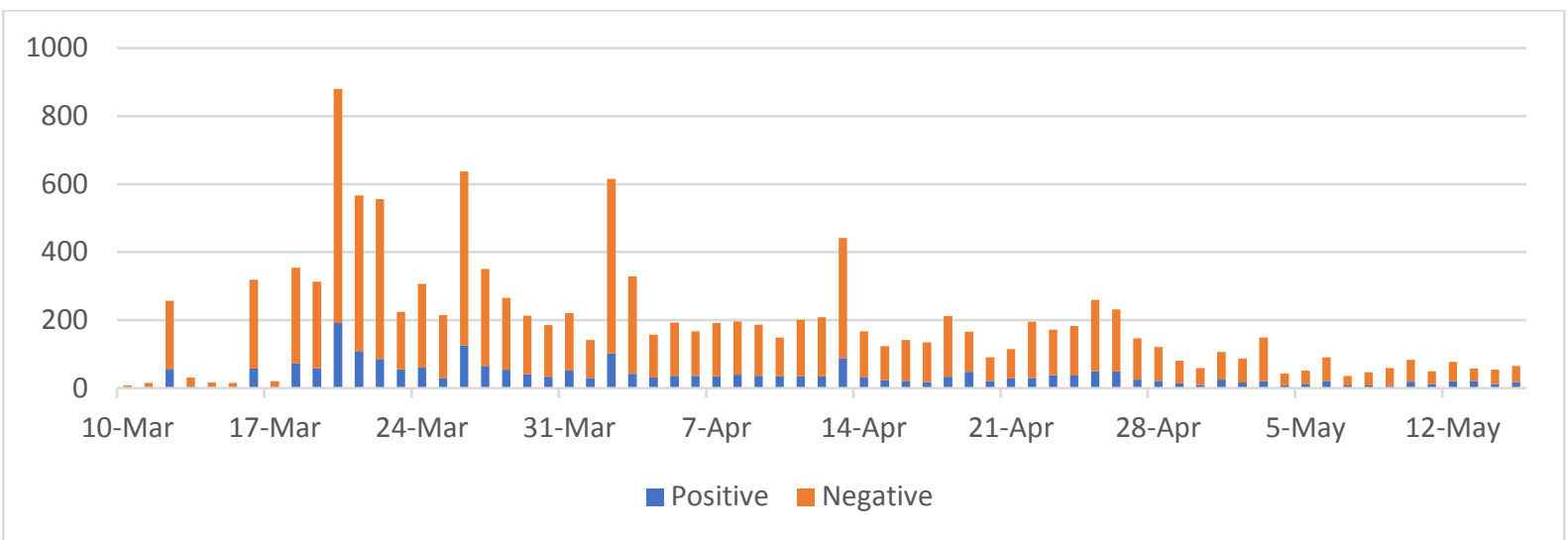

Figure 11 Distribution of Positive and Negative Comments by Days with BERT 


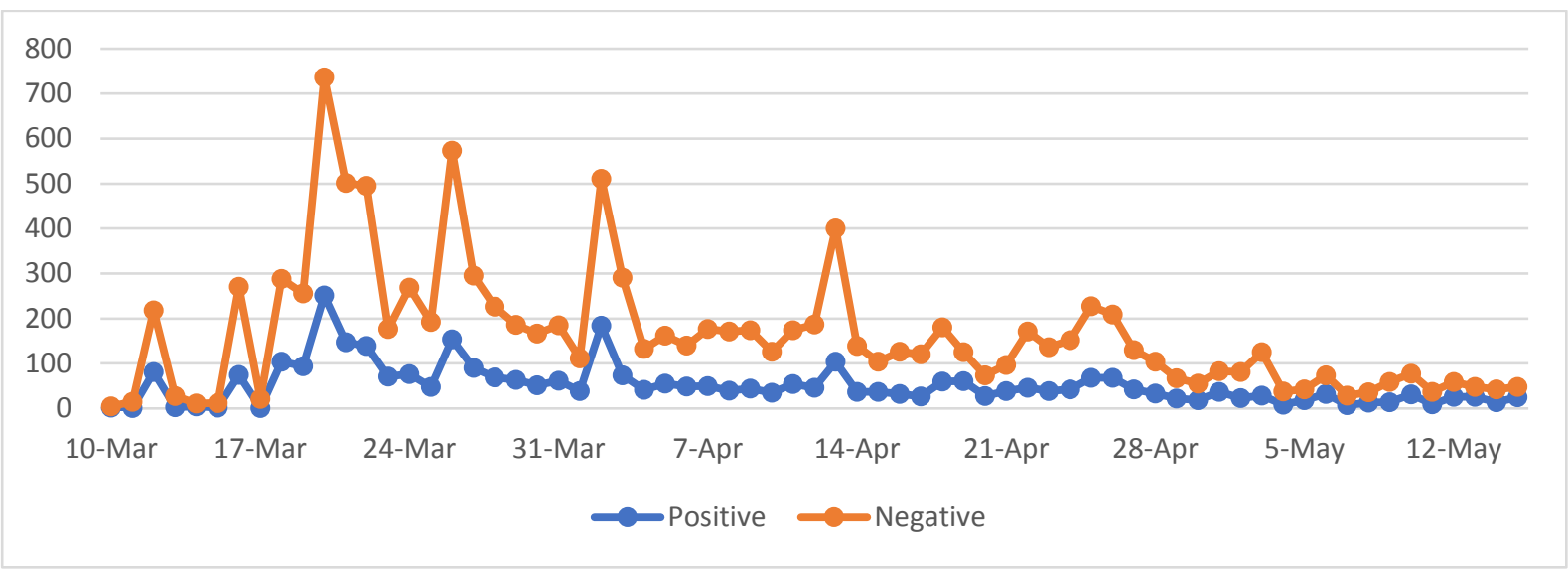

Figure 12 Distribution of Positive and Negative Comments by Days with GRU

Neither an official state statement nor an important event is observed on April 13. However, on April 13, it is seen that the title "When will the COVID-19 end" and the entries related to this title have increased a lot. With the opening of this title, a new virtual discussion environment has been created and people share their ideas in this discussion environment and object to many other ideas.

Although some points are related to the peak points, the reasons why other points are not connected to the peak days are examined. As a result of this review, it is seen that there are more comments on the cases (famous people) who find more place in the society. Again, it is seen that the news, which is regarded as a surprise about the socially anticipated events, received many comments. On the other hand, it can be said that the announcements and explanations about COVID-19 related life and education are less popular since they are realized within the expectations of the users. It seems that issues related to sports and arts associated with COVID-19 almost did not attract any attention during this period.

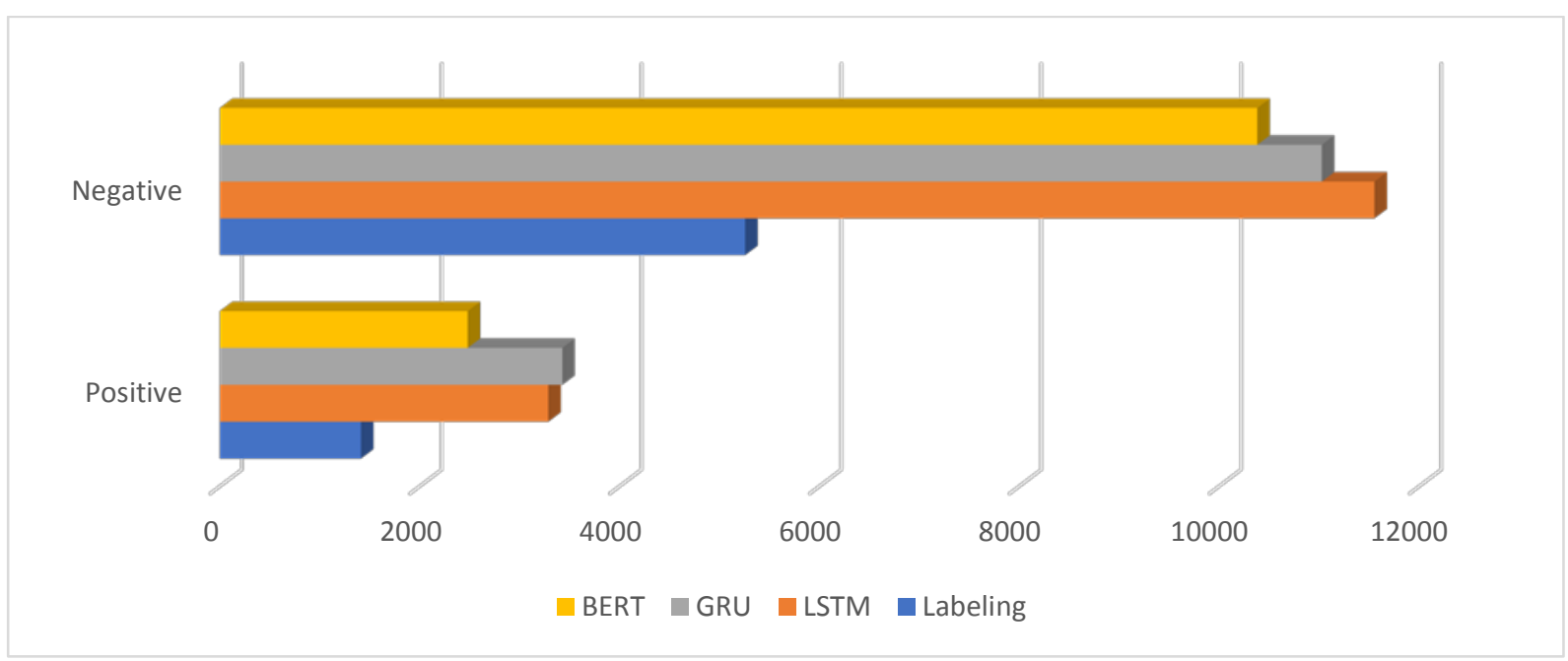

Figure 13 Classification Results of Methods

Figure 13 shows the ratios of positive and negative values of the results of all methods. Neutral values are not shown in Figure 13. It is seen that the success classification results of other methods, except labeling, are close.

\section{Conclusion}

In this study, contents focused on COVID-19 are examined, how emotional the users are affected by current events and the effects of these events on user comments are investigated. More than 10000 comments about COVID-19 collected from online websites between 15 March and 15 May were subjected to 4 different sentiment analysis methods for this purpose. Sentiment analysis and comment frequencies were associated with real events and it was determined how users reacted to which types of 
events. In addition, the connections between the user comments and the number of COVID-19 patients and deaths were determined and shared with the graphics. It has been observed that even if the methods used in the current study do not comply with the language rules, successful results were obtained from irregular data. With the methods will be proposed in the future studies, it is possible to conduct more specific researches in different fields such as government policies, political opinions, the introduction of commercial products and sports competitions. In this way, it will be possible to see the impact of each issue on the process and society in a more tangible way. In line with these data, it will be easier to perceive the priorities and sensitivities of the society in extraordinary situations. In addition, it will be possible to put forward studies for other countries that have or will be affected by the pandemic which they can use as a reference in the process of managing the situation.

\section{References}

[1] K. Ahmed, N. El Tazi, and A. H. Hossny, "Sentiment Analysis over Social Networks: An Overview," in Proceedings - 2015 IEEE International Conference on Systems, Man, and Cybernetics, SMC 2015, pp. 2174-2179, 2015.

[2] D. Guo and C. Chen, "Detecting Non-personal and Spam Users on Geo-tagged Twitter Network,” Trans. GIS, vol. 18, no. 3, pp. 370-384, 2014.

[3] A. K. Uysal and S. Gunal, “The impact of preprocessing on text classification,” Inf. Process. Manag., vol. 50, no. 1, pp. 104-112, 2014.

[4] D. Yang, D. Zhang, Z. Yu, and Z. Wang, "A sentiment-enhanced personalized location recommendation system," in HT 2013 - Proceedings of the 24th ACM Conference on Hypertext and Social Media, pp. 119-128, 2013.

[5] D. Borth, R. Ji, T. Chen, T. Breuel, and S. F. Chang, "Large-scale visual sentiment ontology and detectors using adjective noun pairs," in MM 2013 - Proceedings of the 2013 ACM Multimedia Conference, pp. 223-232, 2013.

[6] M. Anjaria and R. M. R. Guddeti, "A novel sentiment analysis of social networks using supervised learning,” Soc. Netw. Anal. Min., vol. 4, no. 1, pp. 1-15, 2014.

[7] X. Wei, G. Xu, H. Wang, Y. He, Z. Han, and W. Wang, “Sensing Users' Emotional Intelligence in Social Networks,” IEEE Trans. Comput. Soc. Syst., vol. 7, no. 1, pp. 103-112, 2020.

[8] L. Lin, J. Li, R. Zhang, W. Yu, and C. Sun, "Opinion mining and sentiment analysis in social networks: A retweeting structure-aware approach,” in Proceedings - 2014 IEEE/ACM 7th International Conference on Utility and Cloud Computing, UCC 2014, pp. 890-895, 2014.

[9] F. Neri, C. Aliprandi, F. Capeci, M. Cuadros, and T. By, "Sentiment analysis on social media," in Proceedings of the 2012 IEEE/ACM International Conference on Advances in Social Networks Analysis and Mining, ASONAM 2012, pp. 919-926, 2012.

[10] Contratres, F. G., Alves-Souza, S. N., Filgueiras, L. V. L., \& DeSouza, L. S. "Sentiment analysis of social network data for cold-start relief in recommender systems," In Advances in Intelligent Systems and Computing, vol. 746, pp. 122-132, 2018.

[11] Tang, J., Zhang, Y., Sun, J., Rao, J., Yu, W., Chen, Y., \& Fong, A. C. M. “Quantitative study of individual emotional states in social networks", IEEE Transactions on Affective Computing, 3(2), 132-144, 2012. 
[12] F. A. Pozzi, E. Fersini, Sentiment Analysis in Social Networks. Cambridge, M.A, USA: Morgan Kaufmann, 2017.

[13] M. Baldoni, C. Baroglio, V. Patti, and P. Rena, "From tags to emotions: Ontology-driven sentiment analysis in the social Semantic Web," in CEUR Workshop Proceedings, vol. 771, no. 1, pp. 41-54, 2011.

[14] M. Kanakaraj and R. M. R. Guddeti, "NLP based sentiment analysis on Twitter data using ensemble classifiers,” in 2015 3rd International Conference on Signal Processing, Communication and Networking, ICSCN 2015, 2015.

[15] H. Saif, M. Fernandez, Y. He, and H. Alani, "SentiCircles for contextual and conceptual semantic sentiment analysis of Twitter," in Lecture Notes in Computer Science (including subseries Lecture Notes in Artificial Intelligence and Lecture Notes in Bioinformatics), vol. 8465 LNCS, pp. 83-98, 2014.

[16] A. Hasan, S. Moin, A. Karim, and S. Shamshirband, "Machine Learning-Based Sentiment Analysis for Twitter Accounts,” Math. Comput. Appl., vol. 23, no. 1, p. 11, 2018.

[17] V. Sindhwani and P. Melville, "Document-word co-regularization for semi-supervised sentiment analysis," in Proceedings - IEEE International Conference on Data Mining, ICDM, pp. 1025-1030, 2008.

[18] E. Fersini, F. A. Pozzi, and E. Messina, "Approval network: a novel approach for sentiment analysis in social networks,” World Wide Web, vol. 20, no. 4, pp. 831-854, 2017.

[19] A. A. Müngen and M. Kaya, "Influence analysis of posts in social networks by using quadmotifs,” in IDAP 2017 - International Artificial Intelligence and Data Processing Symposium, 2017.

[20] H. Alp, “Çingenelere Yönelik Nefret Söyleminin Ekşi Sözlük’te Yeniden Üretilmesi,” İlef Derg., vol. 3, no. 2, pp. 143-172, 2016.

[21] B. Dogu, B. Dogu, Z. Ziraman, and D. E. Ziraman, "Web Based Authorship in the Context of User Generated Content, An Analysis of a Turkish Web Site: Eksi Sozluk,” Accessed: Oct. 13, 2020 .

[22] F. Akınerdem, "Yerli dizi anlatıları ve izleYici katılımı: uçurum dizisini ekşisÖzlük ve twitter’la birlikte izlemek,” Folklor/Edebiyat, 18(72), pp. 77-90, 2012.

[23] A. Depoux, S. Martin, E. Karafillakis, R. Preet, A. Wilder-Smith, and H. Larson, "The pandemic of social media panic travels faster than the COVID-19 outbreak," Journal of Travel Medicine, Oxford University Press, vol. 27, no. 3, 2020.

[24] J. Gao et al., "Mental health problems and social media exposure during COVID-19 outbreak," PLoS One, vol. 15, no. 4, 2020.

[25] C. Li, L. J. Chen, X. Chen, M. Zhang, C. P. Pang, and H. Chen, "Retrospective analysis of the possibility of predicting the COVID-19 outbreak from Internet searches and social media data, China, 2020," Eurosurveillance, European Centre for Disease Prevention and Control (ECDC), vol. 25, no. 10, 2020.

[26] G. Pennycook, J. McPhetres, Y. Zhang, J. G. Lu, and D. G. Rand, "Fighting COVID-19 Misinformation on Social Media: Experimental Evidence for a Scalable Accuracy-Nudge Intervention,” Psychol. Sci., vol. 31, no. 7, pp. 770-780, 2020. 
[27] L. Li et al., "Characterizing the Propagation of Situational Information in Social Media during COVID-19 Epidemic: A Case Study on Weibo,” IEEE Trans. Comput. Soc. Syst., vol. 7, no. 2, pp. 556-562, 2020.

[28] R. Güner, İ. Hasanoğlu, and F. Aktaş, "COVID-19: Prevention and control measures in community,” TURKISH J. Med. Sci., vol. 50, no. SI-1, pp. 571-577, 2020.

[29] H. Akca, "The Internet as a Participatory Medium: An Analysis of the Eksi Sozluk Website as a Public Sphere,” Theses Diss., [Online]. Available: https://scholarcommons.sc.edu/etd/304, 2010.

[30] “T.C.CUMHURBAŞKANLIĞI : Cumhurbaşkanlığı Sözcüsü Kalın: 'Korona Virüs'le mücadele sürecini, el birliğiyle rehavete ve paniğe kapılmadan atlatma kabiliyetine sahibiz."” https://www.tccb.gov.tr/haberler/410/117021/cumhurbaskanligi-sozcusu-kalin-korona-virus-lemucadele-surecini-el-birligiyle-rehavete-ve-panige-kapilmadan-atlatma-kabiliyetine-sahibiz. accessed Jun. 10, 2020.

[31] “T.C Sağlık Bakanlığı Korona Tablosu.” https://covid19.saglik.gov.tr/ accessed May. 23, 2020.

[32] J. Ding, H. Sun, X. Wang, and X. Liu, "Entity-level sentiment analysis of issue comments,” in Proceedings - International Conference on Software Engineering, pp. 7-13, 2018.

[33] E. Dogan and B. Kaya, "Deep Learning Based Sentiment Analysis and Text Summarization in Social Networks," in 2019 International Conference on Artificial Intelligence and Data Processing Symposium, IDAP 2019, 2019.

[34] M. A. Tocoglu and A. Alpkocak, "TREMO: A dataset for emotion analysis in Turkish,” J. Inf. Sci., vol. 44, no. 6, pp. 848-860, 2018.

[35] T. Mikolov, K. Chen, G. Corrado, and J. Dean, "Efficient estimation of word representations in vector space," in 1st International Conference on Learning Representations, ICLR 2013 Workshop Track Proceedings, 2013.

[36] S. Firat, "GitHub - selimfirat/bilkent-turkish-writings-dataset: Turkish writings dataset that promotes creativity, content, composition, grammar, spelling and punctuation.”. https://github.com/selimfirat/bilkent-turkish-writings-dataset, 2017.

[37] J. Chung, C. Gulcehre, K. Cho, and Y. Bengio, "Empirical Evaluation of Gated Recurrent Neural Networks on Sequence Modeling,” 2014.

[38] R. Rana, “Gated Recurrent Unit (GRU) for Emotion Classification from Noisy Speech,” [Online]. Available: http://arxiv.org/abs/1612.07778, 2016.

[39] J. Devlin, M.-W. Chang, K. Lee, and K. Toutanova, "BERT: Pre-training of Deep Bidirectional Transformers for Language Understanding,” NAACL HLT 2019 - 2019 Conf. North Am. Chapter Assoc. Comput. Linguist. Hum. Lang. Technol. - Proc. Conf., vol. 1, pp. 4171-4186, 2018.

[40] K. Clark, U. Khandelwal, O. Levy, and C. D. Manning, "What Does BERT Look At? An Analysis of BERT’s Attention,” pp. 276-286, 2019.

[41] “Sağlık Bakanı Koca'dan koronavirüs açıklaması: İstanbul Türkiye'nin 'Wuhan'1 oldu - Son Dakika Flaş Haberler." https://www.cnnturk.com/turkiye/saglik-bakani-kocadan-koronavirusaciklamasi-istanbul-turkiyenin-wuhani-oldu, accessed Oct. 13, 2020. 